\section{Editorial for JoCI}

\author{
Vincent R. Bonagura ${ }^{1,2,3}$ - Jean-Laurent Casanova ${ }^{4,5,6,7,8}$
}

Published online: 18 August 2015

(C) Springer Science+Business Media New York 2015

Dear Readers,

We are writing to inform you that we have made several changes in the format of the Journal of Clinical Immunology (JoCI), some of which you may already have noticed over the past months.

We have introduced a new article venue, specifically "continuing medical education (CME) Reviews". Each of these reviews provides a comprehensive description of current knowledge about a single monogenic disorder underlying a primary immunodeficiency (or two or more primary immunodeficiencies, if allelic to the same locus). International experts write these reviews. Readers who wish to obtain CME credit can receive $1 \mathrm{CME}$ credit for each review they have read after completing the 5 question tests that can be found on the Clinical Immunology Society website, see J Clin Immunol (2014) 34:891 for further details of this new program.

Vincent R. Bonagura

VBonagura@NSHS.edu

1 Laboratory for Host Defense, Jeffrey Modell Diagnostic Center, Feinstein Institute for Medical Research, Manhasset, NY, USA

2 Departments of Pediatrics and Internal Medicine, Hofstra NS-LIJ, School of Medicine, Great Neck, NY, USA

3 Alexandra and Steven Cohen Children's Medical Center of New York, North Shore-LIJ Medical Center, Great Neck, NY, USA

4 St. Giles Laboratory of Human Genetics of Infectious Diseases, The Rockefeller University, Rockefeller Branch, New York, NY, USA

5 Howard Hughes Medical Institute, New York, NY, USA

6 Laboratory of Human Genetics of Infectious Diseases, Necker Hospital for Sick Children, Necker Branch, Paris, France

7 Imagine Institute, Paris Descartes University, Paris, France

8 Pediatric Hematology-Immunology Unit, Necker Hospital for Sick Children, Paris, France
We are also initiating a "How I Manage" article program that will also be written by international experts who care for patients with primary immunodeficiencies and related diseases. We envision this program beginning in 2016, as we have invited the first authors who will initiate this program during the summer of 2015 . We are excited by this new program for the JoCI and can assure our readership that these reviews will be cutting edge summaries of varied and relevant topics. The specific description of these two novel types of papers can be found on our website.

Along these lines, we have also redefined and simplified the article types. We will no longer publish "Astute Clinician" and "Brief Scientific Research" reports. There will be only four article types: Letters to the Editor, Original Research, CME Reviews, and How I Manage articles. Importantly the Letters to the Editor may be a very useful venue to report informative case reports, giving enough space to describe the patient, including one table or figure. The format of Original Research articles is maintained. The detailed instructions for authors for each of the four article types is found at http:// www.springer.com/biomed/immunology/journal/10875.

These changes in the JoCI article venues will best serve the JoCI readership and the growing, international community of clinicians and researchers in primary immunodeficiency. Toward this end, we have revised the Aims and Focus of the JoCI: "The Journal of Clinical Immunology publishes high impact papers in the area of human immunology that explore the diagnosis, pathogenesis, prognosis, or treatment of human diseases. The Journal is particularly focused on primary immunodeficiencies and related diseases. These include inborn errors of immunity, in the broad sense of the term, and their underlying diverse phenotypes, including infection, malignancy, allergy, auto-inflammation, and autoimmunity. We consider a wide range of studies in this area, ranging from immunologic assessment, diagnostic approach, prognosis evaluation, 
and treatment intervention. Case reports are also considered if they provide novel findings that advance the field and are accompanied by a succinct review of the relevant medical literature."

Along these lines, to reinforce the international audience of the journal and its focus on primary immunodeficiencies and related diseases, we have recently invited 44 new experts from multiple countries on all continents to join the Editorial Board of the JoCI. They will enrich our existing and outstanding JoCI Editorial Board.

Finally, we believe the time has come to change the cover of the Journal according to this new focus on primary immunodeficiency diseases. While a challenge was issued to the attendees of the last CIS meeting, specifically to submit new designs for consideration for the new JoCI, we now formally announce a request for suggestions on what the cover of JoCI should look like in future. All suggestions from our readers should be sent to our publishing editor, Ms. Rita Beck at Rita.Beck@springer.com, who will forward them on to the Editor in Chief and Deputy Editor for consideration. The win- ning cover submission will entitle the author to free tuition to the next CIS meeting.

We look forward to responses from our readership about our progress and the new venues for the Journal, and we envision the introduction of other new concepts for the JoCI to address the interests of the international community with special interested in primary immunodeficiencies and related diseases.

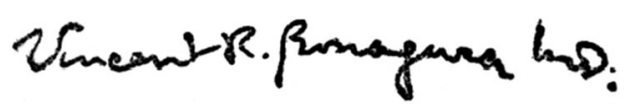

Vincent R. Bonagura MD

Editor in Chief

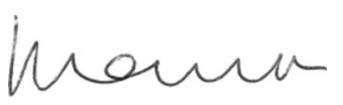

Jean Laurent Casanova MD PhD

Deputy Editor 